\title{
Symptoms and Symptom Clusters in Non Hodgkin's Lymphoma Patients in Turkey
}

\author{
Ferdag Bolukbas ${ }^{1}$, Sevinc Kutluturkan ${ }^{2 *}$
}

\begin{abstract}
Background: Non-Hodgkin's lymphoma (NHL) patients demonstrate multiple symptoms in diagnosis and treatment processes. This cross-sectional descriptive study aimed to determine the symptoms and symptom clusters in such patients receiving chemotherapy. Materials and Methods: The study was carried out on a total of 110 inpatients and outpatients receiving treatment in $\mathbf{7}$ hospitals in Ankara, Turkey. A questionnaire form and the Memorial Symptom Assessment Scale (MSAS) were applied. Percentages, means, $t$ test, one way analysis of variance (ANOVA) and cluster analysis were used for statistical analyses. Results: The most prevalent symptoms in this study were lack of energy, hair loss and change in the way food tastes. The most severe symptoms were hair loss, change in the way food tastes and constipation. The top three most distressing symptoms were taste change, constipation and mouth sores. Seven symptom clusters were determined in this study. Conclusions: Findings of the study will provide an understanding of symptom experiences of Non-Hodgkin's lymphoma patients receiving chemotherapy and will guide determining appropriate nursing interventions. Receiving care of desired quality will contribute to increasing quality of life of affected individuals.
\end{abstract}

Keywords: Non Hodgkin's lymphoma - symptom clusters - treatment - memorial symptom assessment scale

Asian Pac J Cancer Prev, 15 (17), 7153-7158

\section{Introduction}

Individuals experience a wide range of symptoms in case of an illness during the process of diagnosis and treatment. The symptoms which occur during the treatment process interact with each other and form symptom clusters. Dood et al. (2009), defined symptom cluster as "the case which results from the occurrence and interaction of three or more symptoms".

Occurrence of the symptoms, which form symptom clusters, are affected from physical factors such as type, stage and treatment of disease; psychosocial factors such as marital status, gender, anxiety, depression and habits. It was reported that physiological, psychological and social factors cause occurrence of more than one symptom or more severe perception of the existing symptom (Lenz et al., 1997; Barsevick, 2007; Kirkova et al., 2007).

Individual or collective effects of these factors, which affect the occurrence of the symptoms forming symptom clusters and their intensity in a cluster, on symptom clusters should be understood. A review of the literature found that symptom clusters have been investigated in specific diseases such as breast and brain tumor or in heterogeneous groups such as childhood cancers (Okuyama et al., 2003; Chen and Tseng, 2005; Walsh and Rybicki, 2006; Cheung and Zimmermann, 2009; Henoch et al., 2009; Yamagishi et al., 2009; Molassiotis et al.,
2010; Atay et al., 2012; Erickson et al., 2013).

However, it's recommended that symptom cluster studies should concentrate on only one type of cancer rather than heterogeneous groups (Gift, 2007). Analysis of symptom clusters on a certain disease group will enable prevention of symptoms and determining effective nursing interventions for the management of symptoms by enabling the understanding of synergic characteristics of the symptoms which develop due to the disease or the treatment (Paice, 2004; Given et al., 2007).

Symptom clusters have a significant impact on functional, emotional state and quality of life of the patients in case of a disease such as cancer and makes symptom control difficult in patient care (Ferreira et al., 2008; Maliski et al., 2008; Dodd et al., 2010; Dodd et al., 2011). NHL is observed as the 7 th most common type of cancer in males and 9th most common type of cancer in females in the Turkey (Eser et al., 2010). An increase in the NHL incidence was found (Yildırım et al., 2013). Both literature data and our clinical experience indicate that among cancer patients, Non-Hodgkin's lymphoma (NHL) patients encounter multiple symptoms in diagnosis and treatment process, which might be difficult to control. The patients suffer from the adverse effects of chemotherapy, which is the most commonly used treatment approach. Despite this, a review of the literature found no specific research on the symptom cluster of NHL patients. In fact, 
symptom management, which has a significant role in nursing care of NHL patients, is of great importance for the nurses to determine symptom clusters and to apply necessary nursing interventions.

Therefore, this study will enable determination of symptom clusters, which play a significant role in effective symptom management and identification of symptom frequency, severity and distress levels as the elements which make understanding of a symptom experience of the individual easier. We believe that developing nursing interventions for symptom clusters will decrease the symptom load of the individuals; positively affect physical and psychosocial functions of the patients and contribute to improving quality of life.

The aim of this study is to determine frequency and severity of symptoms, distress level and symptom clusters experienced by NHL patients receiving chemotherapy.

\section{Materials and Methods}

\section{This is a cross-sectional descriptive study}

Study questions: What are the frequency and severity of symptoms and distress levels experienced in NonHodgkin's lymphoma patients following chemotherapy?

Do the socio-demographic variables have an impact on the symptoms experienced by Non-Hodgkin's lymphoma patients following chemotherapy?

What are the symptom clusters experienced by NonHodgkin's lymphoma patients following chemotherapy?

This cross-sectional descriptive study was carried out in 7 hospitals in Ankara of Turkey in December 2010-March 2011. A total of 110 inpatients and outpatients in these centers were included in the study. Inclusion criteria included age 18 years and older, NHL diagnosis, receiving chemotherapy, being able to communicate and voluntariness.

Written permission was taken from Gazi University Medical Faculty Institutional Review Board AnkaraTurkey (June 25 2010 Ethical Committee Protocol \# 083) and related departments for the study. The study was carried out on voluntary basis and in accordance with the principles of Helsinki Declaration. Individuals who were included in the study were informed about the study and their consents were taken.

The patients who met inclusion criteria were determined through patient registration system and the interviews with the doctor and nurses in the clinic. The forms were collected by the researcher through faceto-face interviews in one session. The interviews were performed in polyclinic room in out-patient clinic and in patients' rooms in the clinics. An interview lasted for 35-40 minutes on average. Questionnaire form and Memorial Symptom Assessment Scale (MSAS) was used to collect data.

Questionnaire form consisted of a total of 24 questions, 17 of which aimed to determine sociodemographic characteristics of the patients such as age, gender, educational status and having chronic diseases. 7 questions aimed to determine characteristics on disease and treatment such as stage of disease, treatment protocol and number of cure.
Memorial Symptom Assessment Scale (MSAS): The scale was developed by Portenoy et al. (1994). MSAS is a 32-item multidimensional scale and is used to evaluate the symptoms experienced by cancer patients in the last one week. The scale has three sub-dimensions involving the frequency and severity and the symptom distress of 24 symptoms in the last one week; 8 symptoms have two subdimensions involving the severity and symptom distress. "Frequency" and "severity" levels of the symptoms are responded in 4-item Likert type scale while "distress" levels are responded in 5-item Likert type scale (Y1ldırım et al., 2011).

Validity and reliability study of MSAS in Turkish found that the scale has moderately high internal consistency reliability in subscales and in general of the scale Cronbach's alpha ranged from 0.71-0.84. The scale had a test-re-test reliability of 0.78 (Y1ldırım et al., 2011). In our study, Cronbach's alpha coefficient for MSAS scale was 0.89 .

Statistical Package For Social Science 15 (SPSS Inc., Chicago, IL, USA.) package program was used for data analysis. Frequency and percentages were calculated. ANOVA (F) test was used to compare number of symptoms concerning age, educational status, occupation, disease stage, treatment period and number of received cures. T test was used to compare number of symptoms concerning gender, marital status, having chronic diseases, smoking and alcohol consumption and received drug protocol.

Software Multi-Variate Statistical Package (MVSP) v.3.12 (Pearson Coefficient, Further Nearest) was used to determine symptom clusters (Kovach, 2007). There is no consensus in determining symptom clusters, on the number of symptoms which will be included in the analysis. The symptoms with a prevalence of below $20 \%$ were excluded from this study. Kim et al. (2005), defined symptom cluster as "occurrence and interaction of two or more symptoms." This study sought the concurrence of at least two symptoms to form symptom clusters. Symptom clusters were determined by considering Pearson's correlation coefficient as $25 \%$ and above.

\section{Results}

\section{Demographic and clinical characteristics}

Data on the demographic and clinical characteristics of 110 patients receiving chemotherapy, who were followed up with NHL diagnosis are presented in Table 1.

\section{Symptom clusters}

A total of 7 symptom clusters were determined in NHL patients receiving chemotherapy (Figure 1).

Cluster 1: mouth sores, dry mouth

Cluster 2: dizziness, difficulty swallowing, shortness of breath

Cluster 3: pain, difficulty sleeping, constipation, weight loss, sweats

Cluster 4: nausea, vomiting, lack of appetite, diarrhea

Cluster 5: feeling bloated, cough, feeling sad, difficulty concentrating

Cluster 6: changes in the skin, feeling irritable, feeling 
Table 1. Demographic and Clinical Characteristics

\begin{tabular}{|c|c|c|}
\hline \multicolumn{2}{|l|}{ Characteristics } & $\%$ \\
\hline \multicolumn{3}{|c|}{ Age $($ mean \pm SD) $($ mean 46.91 \pm 16.99$)(\min : 20$ max: 77) } \\
\hline \multirow[t]{2}{*}{ Gender } & Male & 60.9 \\
\hline & Female & 39.1 \\
\hline \multirow[t]{5}{*}{ Education level } & Illiterate & 13.6 \\
\hline & Literate & 2.7 \\
\hline & Primary and Junior High School & 46.4 \\
\hline & Senior High school & 14.6 \\
\hline & University & 22.7 \\
\hline \multirow[t]{2}{*}{ Marital status } & Married & 80.9 \\
\hline & Single & 19.1 \\
\hline \multirow[t]{2}{*}{ Chronic illness } & Yes & 51.8 \\
\hline & No & 48.2 \\
\hline \multirow[t]{2}{*}{ Smoking } & Smoker & 50.0 \\
\hline & Non-smoker & 50.0 \\
\hline \multirow[t]{2}{*}{ Alcohol consumption } & Consumes alcohol & 13.6 \\
\hline & Doesn't consume alcohol & 86.4 \\
\hline \multirow[t]{4}{*}{ Clinical stage } & Stage I & 4.5 \\
\hline & Stage -II & 6.4 \\
\hline & Stage -III & 39.1 \\
\hline & Stage -IV & 50.0 \\
\hline \multirow[t]{4}{*}{ Previous treatments } & Chemotherapy & 82.7 \\
\hline & Chemotherapy _ surgery & 3.7 \\
\hline & Chemotherapy -radiotherapy & 11.8 \\
\hline & Chemotherapy - radiotherapy - su & y 1.8 \\
\hline \multirow{4}{*}{$\begin{array}{l}\text { Duration of treatment } \\
\text { (month) }\end{array}$} & $1-3$ & 41.8 \\
\hline & $4-6$ & 32.7 \\
\hline & $7-9$ & 13.7 \\
\hline & 10 and longer & 11.8 \\
\hline \multirow[t]{2}{*}{ Type of current treatment } & RCHOP & 79.1 \\
\hline & RDHAP & 20.9 \\
\hline \multirow[t]{3}{*}{ Current number of cure } & 1-3.cure & 45.5 \\
\hline & 4-6.cure & 36.4 \\
\hline & 7 and higher cure & 18.1 \\
\hline
\end{tabular}

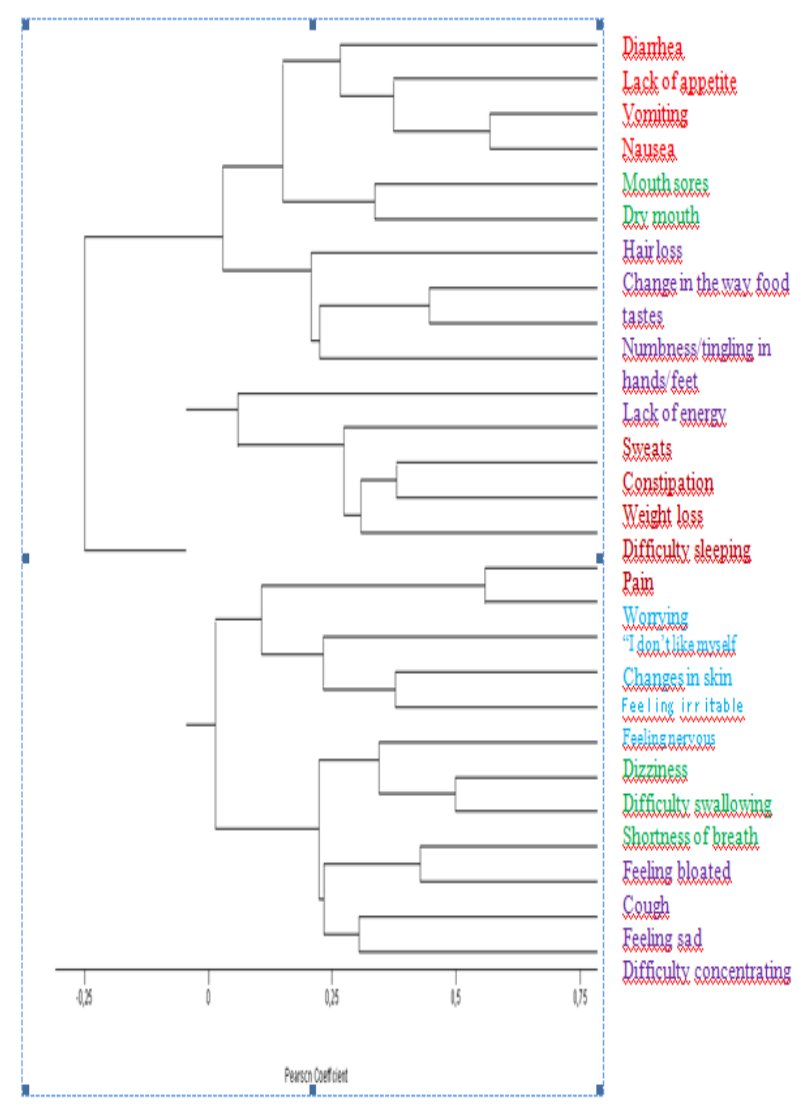

Figure 1. Symptom Clusters were Determined in NHL Patients Receiving Chemotherapy. Frequency and severity of symptoms and distress levels

Table 2. Symptom Prevalence. Symptom frequency. Symptom Severity and Symptom Distress

\begin{tabular}{|c|c|c|c|c|c|c|c|}
\hline \multirow[t]{2}{*}{ Symptom } & \multirow[t]{2}{*}{ Symptom prevalance $(\%)$} & \multicolumn{2}{|c|}{ Symptom frequency } & \multicolumn{2}{|c|}{ Symptom Severity } & \multicolumn{2}{|c|}{ Symptom Distress } \\
\hline & & Mean & Std. Deviation & Mean & Std. Deviation & Mean & Std. Deviation \\
\hline Lack of energy (n:102) & 92.7 & 3.14 & 0.833 & 2.38 & 0.704 & 2.06 & 1.115 \\
\hline Hair loss (n:96) & 87.3 & - & - & 3.06 & 0.844 & 2.04 & 1.486 \\
\hline Change in the way food tastes (n:86) & 77.3 & - & - & 2.92 & 0.923 & 2.72 & 1.195 \\
\hline Dry mouth (n:84) & 76.4 & 3.07 & 0.954 & 2.45 & 0.767 & 2.02 & 1.161 \\
\hline Feeling irritable(n:83) & 75.5 & 2.83 & 0.730 & 2.41 & 0.645 & 1.78 & .938 \\
\hline Feeling Nervous (n:81) & 73.6 & 2.81 & 0.910 & 2.43 & 0.757 & 1.74 & 1.093 \\
\hline Nausea (n:77) & 70.0 & 2.30 & 0.988 & 2.12 & 0.725 & 1.91 & 1.258 \\
\hline Feeling sad (n:77) & 70.0 & 2.71 & 0.871 & 2.40 & 0.748 & 1.91 & .934 \\
\hline Mouth sores (n:77) & 70.0 & - & - & 2.38 & 0.932 & 2.34 & 1.108 \\
\hline Lack of appetite (n:73) & 66.4 & 2.90 & 0.988 & 2.59 & 0.831 & 2.16 & 1.247 \\
\hline Feeling drowsy (n:73) & 66.4 & 2.53 & 0.835 & 2.26 & 0.746 & 1.64 & 1.085 \\
\hline Constipation (n: 71) & 64.5 & - & - & 2.70 & 0.901 & 2.51 & 1.170 \\
\hline Numbness/tingling in hands/feet (n: 71) & 63.6 & 3.03 & 0.971 & 2.28 & 0.796 & 1.90 & 1.110 \\
\hline Sweats (n: 66) & 60.0 & 2.62 & 0.907 & 2.23 & 0.740 & 1.50 & .996 \\
\hline Worrying (n: 66) & 60.0 & 2.92 & 0.865 & 2.48 & 0.881 & 1.94 & 1.051 \\
\hline Difficulty concentrating (n: 62) & 56.4 & 2.24 & 0.935 & 1.98 & 0.665 & 1.27 & .978 \\
\hline Pain (n: 59) & 53.6 & 2.86 & 0.860 & 2.29 & 0.720 & 2.08 & .970 \\
\hline Difficulty sleeping (n: 59) & 53.6 & 2.78 & 0.789 & 2.36 & 0.783 & 2.00 & 1.067 \\
\hline Changes in skin (n: 59) & 53.6 & -- & - & 2.32 & 0.880 & 1.76 & 1.194 \\
\hline Weight loss (n: 57) & 51.8 & - & - & 2.18 & 0.782 & 1.65 & 1.232 \\
\hline Vomiting (n: 51) & 46.4 & 2.00 & 0.980 & 2.04 & 0.871 & 1.76 & 1.210 \\
\hline "I don’t look like myself” (n: 50) & 45.5 & & & 2.68 & 0.913 & 2.34 & 1.154 \\
\hline Difficulty swallowing (n: 41) & 37.3 & 2.05 & 0.773 & 1.83 & 0.704 & 1.68 & .986 \\
\hline Feeling bloated (n: 41) & 37.3 & 2.59 & 0.948 & 2.29 & 0.716 & 1.76 & 1.067 \\
\hline Diarrhea (n: 38) & 34.5 & 2.18 & 0.865 & 2.08 & 0.673 & 1.53 & 1.059 \\
\hline Cough (n38: ) & 34.5 & 1.79 & 0.741 & 1.74 & 0.760 & 1.08 & .997 \\
\hline Dizziness (n: 36) & 32.7 & 1.97 & 0.845 & 1.83 & 0.775 & 1.61 & 1.103 \\
\hline Shortness of breath (n: 28) & 25.5 & 2.14 & 0.932 & 1.86 & 0.651 & 1.71 & 1.117 \\
\hline Swelling of arms or legs (n: 27) & 24.5 & - & - & 2.19 & 0.786 & 1.85 & 1.167 \\
\hline Itching (n: 24) & 21.8 & 2.21 & 1.021 & 2.04 & 0.859 & 1.50 & 1.180 \\
\hline Problems with sexual interest or activity (n: 20) & 18.2 & 2.35 & 1.137 & 2.35 & 0.988 & 2.05 & 1.504 \\
\hline Problems with urination (n: 16) & 14.5 & 2.19 & 1.167 & 2.19 & 0.911 & 1.81 & 1.377 \\
\hline
\end{tabular}


nervous, worrying "I don't look like myself".

Cluster 7: hair loss, change in the way food tastes, numbness/tingling in hands/feet, lack of energy.

Our results on the frequency and severity of symptoms and the distress level as the factors which provide a complete understanding of a symptom experience on the individuals are:

The symptoms with the highest prevalence among patients were found to be lack of energy, hair loss, change in the way food tastes, dry mouth, feeling irritable, feeling nervous, mouth sores, feeling sad and nausea.

The most frequent symptoms were found to be lack of energy followed by dry mouth, numbness/tingling in hand/feet, worrying and lack of appetite.

Top five most severe symptoms experienced by the patients were found to be hair loss followed by change in the way food tastes, constipation, "I don't look like myself" and lack of appetite.

Top five symptoms which caused the most distress in patients were found to be change in the way food tastes, constipation, mouth sores, "I don't look like myself" and lack of appetite (Table 2).

Comparison of the symptoms experienced by the patients due to chemotherapy according to their sociodemographic and clinical characteristics revealed the following.

It was found that there was no significant difference between the number of symptoms experienced by patients and their socio-demographic characteristics $(p>0.05)$.

Comparison of the number of symptoms experienced by the patients with their medical conditions showed that the patients having another chronic disease apart from cancer experienced significantly more symptoms $(\mathrm{t}=3.619, p<0.05)$.

\section{Discussion}

Symptom cluster was not specifically studied in NHL patients. In this study, we studied symptom cluster of outpatient and inpatient NHL patients who specifically received chemotherapy. A total of 7 symptom clusters were determined in this study (Graph 1).

Mouth sores and dry mouth formed a cluster in our study. Concurrence of these two symptoms was considered as an expected result in terms of the interaction of symptoms. In a study carried out by Okuyama et al., dry mouth alone formed a cluster, while the study of Walsh and Rybicki, dry mouth; easy fatigue, weakness, anorexia, lack of energy, early satiety, weight loss, taste changes formed a cluster (Okuyama et al., 2003; Walsh and Rybicki, 2006).

In our study, second cluster included dizziness, difficulty swallowing and dyspnea. Dyspnea and dizziness were considered as related symptoms and dysphagia was added to this cluster. Similar to our study, dysphagia and dyspnea were added to the same cluster in the study of Walsh and Rybicki. Only cough and hoarseness were added to this cluster (Walsh and Rybicki, 2006).

In our study, pain, difficulty sleeping and constipation were included in the same cluster. Similarly, pain and constipation formed a single cluster in the study of Walsh and Rybicki, while pain, fatigue, sleep disturbance, lack of appetite, and drowsiness formed a cluster in the study of Chen and Tseng. (Chen and Tseng, 2005; Walsh and Rybicki 2006). In a study carried out by Henoch et al. (2009), bowel issues, nausea, appetite loss and fatigue formed a cluster.

In our study, among gastrointestinal symptoms, nausea, vomiting, lack of appetite, diarrhea formed the 4. cluster. Similar to our study, literature data showed that gastrointestinal symptoms were included in the same cluster, while two or three of these symptoms formed a cluster. In the studies, different symptoms such as pain and fatigue, drowsiness, dyspnea, constipation were added in the same cluster (Okuyama et al., 2003; Chen and Tseng, 2005; Cheung et al., 2009; Henoch et al., 2009; Yamagishi et al., 2009).

In 7 symptom clusters in our study, physical and psychosocial symptoms were included in the same cluster excluding a few clusters. As stated in the Theory of Unpleasant Symptoms (TOUS), which affects the understanding of the concept of symptom clusters, a synergic interaction takes place between the symptoms when they exist at the same time.

A symptom causes occurrence or more severe perception of another symptom (Lenz et al., 1997). In our study, feeling bloated and cough in the 5. cluster and changes in skin symptom in the 6 . cluster were included in the same cluster with psychological symptoms and indicate the synergic interaction between the symptoms.

In our study, the symptoms which are not considered to be related with each other in the 7 . cluster were included in the same cluster. We believe that change in the way food tastes symptom in this cluster was related with lack of energy symptoms. Taste changes and lack of energy were included in the same cluster in the study of Walsh and Rybicki. In a study carried out by Molassiotis et al., numbness/tingling in hand/feet, swelling of arms/legs were included in a cluster(Walsh and Rybicki, 2006; Molassiotis et al., 2010).

Based on the collective analysis of the results of our study and literature data, it is believed that sociodemographic and clinical characteristics of the patients in occuring symptom clusters caused concurrence of different symptoms in the same cluster. This indicates the physical, social and psychological factors, which determine a symptom experience, should be analyzed in terms of an effective symptom management.

\section{Symptom characteristics}

Individuals who are diagnosed with cancer experience various disease and treatment related symptoms. It was found that the patients experienced varying degrees of potential problems in the treatment protocol they receive. Previous studies on cancer patients showed that the most common symptoms were pain, weight loss, dry mouth , lack of appetite, sweats , fatigue, lack of energy, difficulty sleeping, feeling drowsy and sweats, hair loss, nervousness (Yan and Sellick, 2004; Chen and Tseng, 2005; Ogce and Ozkan, 2008; Kim et al., 2009; Nazik et al., 2012; Siefert, 2010). These results are similar to our findings.

Prevalence of a symptom alone is not adequate to determine the effects of a symptom experience on 
individuals. Severity and distress levels, which are other elements of symptom experience should also be determined. Similar to our findings, studies on cancer patients showed that the most severe symptoms were hair loss, change in the way food tastes, decreased appetite, dry mouth, constipation and sadness. Unlike our findings, the most severe symptoms were shortness of breath, fatigue, nausea, vomiting, weight loss, insomnia, somnolence, stomach pain, pain and decreased sex interest (Wang et al., 2002; Yan and Sellick, 2004; Chen and Tseng, 2005; Cheung et al., 2009; Yamagishi et al., 2009; Ryu et al., 2010; Williams et al., 2010). It is believed that the similarity and difference between the literature data and our study result from difficult sampling groups used in the studies.

In our study, change in the way food tastes, constipation, mouth sores and "I don't look like myself" symptoms were found to be the most distressing symptoms. We believe that this result is caused by the fact that these symptoms were experienced more frequently and had higher severity values. Similar to our study, in a study carried out by Yan and Sellick, the most distressing symptoms were reported to be change in the way food tastes, while different symptoms such as insomnia, hair loss, fatigue, shortness of breath, itching, cough and weight loss were other most distressing symptoms (Yan and Sellick, 2004). Unlike our study, Cooley et al. (2003) carried out a study on lung cancer patients who received chemotherapy and reported that fatigue and pain were the most distressing symptoms (Cooley et al., 2003).

We believe that the result on the frequency, severity and distress levels of symptoms was related with individual characteristics of patients, different stages of disease, treatment protocol they receive and number of cures.

Furthermore, there is no consistency between prevalence of symptoms and frequency, severity an distress levels. Although a symptom has a high prevalence ratio, its frequency or severity is not as high (Yan and Sellick, 2004). This indicates that in effective symptom control studies, in addition to the prevalence of a symptom, frequency, severity and distress dimensions, which showed the effects of a symptom on the individual, should also be taken into account.

Cancer patients experience various symptoms due to disease and treatment process. It is important to know the symptoms and symptom clusters experienced in specific disease groups to achieve an effective and accurate symptom control.

Results of our study determined the prevalence, period, distress levels and symptom clusters due to chemotherapy in a group with the same diagnosis at varying treatment stages. Based on these results, determining the symptoms which should be primarily analyzed in care process of NHL patients will enable identification of effective interventions by the understanding of symptom experiences. In conclusion, an effective symptom management in patients with NHL will contribute to increasing quality of life of individuals.

\section{Acknowledgements}

We would like to thank participants and the hospital administrative.

\section{References}

Atay S, Conk Z, Bahar Z (2012). Identifying symptom clusters in paediatric cancer patients using the Memorial Symptom Assessment Scale. Eur J Cancer Care, 21, 460-8.

Barsevick AM (2007). The concept of symptom cluster. Semin Oncol Nurs 23, 89-98.

Chen ML, Tseng HH (2005). Identification and verification of symptom clusters in cancer patients. J Support Oncol, 3, 28-9.

Cheung WY, Le LW, Zimmermann C (2009). Symptom clusters in patients with advanced cancers. Support Care Cancer, 17, 1223-30.

Cooley ME, Short TH, Moriarty HJ (2003). Symptom prevalence, distress, and change over time in adults receiving treatment for lung cancer. Psychooncology, 12, 694-708.

Dood MJ, Miaskowski C, Lee KA (2009). Occurrence of symptom clusters. J Natl Cancer Inst Monographs, 32, 76-78.

Dodd MJ, Cho MH, Cooper BA, Miaskowski C (2010). The effect of symptom clusters on functional status and quality of life in women with breast cancer. Eur J Oncol Nurs, 14, 101-10.

Dodd MJ, Cho MH, Cooper BA, et al (2011). Identification of latent classes in patients who are receiving biotherapy based on symptom experience and its effect on functional status and quality of life. Oncol Nurs Forum, 38, 33-42.

Erickson JM, Macpherson CF, Ameringer S, et al (2013). Symptoms and symptom clusters in adolescents receiving cancer treatment: A review of the literature. Int J Nurs Stud, 50, $847-69$.

Eser S, Yakut C, Ozdemir R, et al (2010). Cancer incidence rates in Turkey in 2006: a detailed registry based estimation. Asian Pac J Cancer Prev, 11, 1731-9.

Ferreira KA, Kimura M, Teixeira MJ, et al (2008). Impact of cancer-related symptom synergisms on health-related quality of life and performance status. J Pain Symptom Manage, 35, 604-16.

Gift AG (2007). Symptom clusters related to specific cancers. Semin Oncol Nurs, 23, 136-41.

Given BA, Given CW, Sikorskii A, Hadar N (2007). Symptom clusters and physical function for patients receiving chemotherapy. Semin Oncol Nurs, 23, 121-6.

Henoch I, Ploner A, Tishelman C (2009). Increasing stringency in symptom cluster research: a methodological exploration of symptom clusters in patients with inoperable lung cancer. Oncol Nurs Forum, 36, 282-92.

Kim HJ, McGuire DB, Tulman L, Barsevick AM (2005). Symptom clusters: concept analysis and clinical implications for cancer nursing. Cancer Nurs, 28, 270-82.

Kim E, Jahan T, Aouizerat BE, et al (2009). Changes in symptom clusters in patients undergoing radiation therapy. Support Care Cancer, 17, 1383-91.

Kirkova J, Walsh D, Aktas A, Davis MP (2010). Cancer symptom clusters: old concept but new data. Am J Hosp Palliat Care, 27, 282-8.

Kovach, WL (2007). MVSP-A MultiVariate Statistical Package for Windows, ver. 3.1. Kovach Computing Services, Pentraeth, Wales, U.K.

Lenz ER, Pugh LC, Milligan RA, Gift A, Suppe F (1997). The Middle-Range Theory Of Unpleasant Symptoms: An 
Update. Adv Nursing Sci, 19, 14-27.

Maliski SL, Kwan L, Elashoff D, Litwin MS (2008). Symptom clusters related to treatment for prostate cancer. Oncol Nurs Forum, 35, 786-93.

Molassiotis A, Wengström Y, Kearney N (2010). Symptom cluster patterns during the first year after diagnosis with cancer. J Pain Symptom Manage, 39, 847-58.

Nazik E, Arslan S, Nazik H, et al (2012). Anxiety and symptom assessment in Turkish gynecologic cancer patients receiving chemotherapy. Asian Pac J Cancer Prev, 13, 3129-33.

Okuyama T, Wang XS, Akechi T, et al (2003). Japanese version of the MD Anderson Symptom Inventory: a validation study. J Pain Symptom Manage, 26, 1093-104.

Ogce F, Ozkan S (2008). Changes in functional status and physical and psychological symptoms in women receiving chemotherapy for breast cancer. Asian Pac J Cancer Prev, 9, 449-52.

Paice JA (2004). Assessment of symptom clusters in people with cancer. J Natl Cancer Inst Monogr, 32, 98-102.

Portenoy RK, Thaler HT, Kornblith AB, et al (1994). The Memorial Symptom Assessment Scale: an instrument for the evaluation of symptom prevalence, characteristics and distress. Eur J Cancer, 30, 1326-36.

Ryu E, Kim K, Cho MS, et al (2010). Symptom clusters and quality of life in Korean patients with hepatocellular carcinoma. Cancer Nurs, 33, 3-10.

Siefert ML (2010). Fatigue, pain, and functional status during outpatient chemotherapy. Oncol Nurs Forum, 37, 114-23.

Walsh D, Rybicki L (2006). Symptom clustering in advanced cancer. Support Care Cancer, 14, 831-6.

Wang XS, Giralt SA, Mendoza TR, et al (2002). Clinical factors associated with cancer-related fatigue in patients being treated for leukemia and non-Hodgkin'slymphoma. J Clin Oncol, 20, 1319-28.

Williams PD, Balabagno AO, Manahan L, et al (2010). Symptom monitoring and self-care practices among Filipino cancer patients. Cancer Nurs, 33, 37-46.

Yamagishi A, Morita T, Miyashita M, Kimura F (2009). Symptom prevalence and longitudinal follow-up in cancer outpatients receiving chemotherapy. J Pain Symptom Manage, 37, 823-30.

Yan H, Sellick K (2004). Symptoms, psychological distress, social support, and quality of life of Chinese patients newly diagnosed with gastrointestinal cancer. Cancer Nurs, 27, 389-99.

Yıldırım Y, Tokem Y, Bozkurt N, et al (2011). Reliability and validity of the Turkish version of the Memorial Symptom Assessment Scale in cancer patients. Asian Pac J Cancer Prev, 12, 3389-96.

Yıldırım M, Karakılınc H, Yıldız M, et al (2013). Non-Hodgkin lymphoma and pesticide exposure in Turkey. Asian Pac J Cancer Prev, 14, 3461-3. 